\title{
The Effect of High-Power and Low-Power Lasers on Symptoms and the Nerve Conduction Study in Patients With Carpal Tunnel Syndrome. A Prospective Randomized Single-Blind Clinical Trial
}

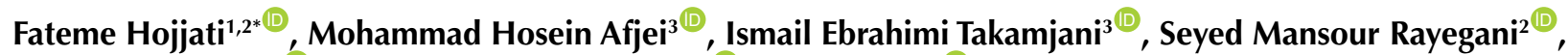 \\ Javad Sarrafzadeh ${ }^{3}{ }^{\circledR}$, Seyed Ahmad Raeissadat ${ }^{2,4}{ }^{\circledR}$, Sara Payami ${ }^{\circledR}{ }^{\circledR}$
}

'Laser Application in Medical Sciences Research Center, Shahid Beheshti University of Medical Sciences, Tehran, Iran ${ }^{2}$ Physical medicine \& rehabilitation research center, Shahid Beheshti University Of Medical Sciences, Tehran, Iran ${ }^{3}$ Department of Physiotherapy, School of Rehabilitation Sciences, Iran University of Medical Sciences and Health Services (IUMS), Tehran, Iran

${ }^{4}$ Clinical Development Research Center of Shahid Modarres Hospital, Shahid Beheshti University of Medical Sciences, Tehran, Iran

${ }^{5}$ Department of Emergency Medicine, Amir al momenin Hospital, Tehran Azad University of Medical Sciences, Tehran, Iran

\section{*Correspondence to} Fateme Hojjati,Laser Application in Medical Sciences Research Center, Shahid Beheshti University of Medical Sciences, Tehran, Iran

Physical medicine \&

rehabilitation research center Shahid Beheshti University Of Medical Sciences, Tehran, Iran. Email:Fatemehojjati@yahoo.com

Published online December 30 2020

\begin{abstract}
Introduction: Carpal tunnel syndrome (CTS) is the most common peripheral nerve entrapment. Various treatments have been suggested for CTS and there is no consensus on their superiority and the order of their use. Laser therapy is a non-invasive treatment method for many musculoskeletal diseases, including CTS. This study aimed to determine and compare the effect of high-power lasers (HPLs) and low-power lasers (LPLs) on pain severity, function, pinch strength, and nerve conduction study findings in patients aged 30-50 years with mild or moderate CTS.

Methods: This is a prospective, randomized, single-blind clinical trial. The study population included 45 patients aged 30-50 years who came to the physical medicine and rehabilitation clinic of Shohada-e-Tajrish hospital and mild or moderate CTS was confirmed for them. Patients were randomly assigned to control, LPL therapy, and HPL therapy groups. Pain, function, pinch strength, and nerve conduction study findings were recorded in all groups before, immediately and 12 weeks after the treatment. All data were compared using SPSS version 21.

Results: All groups showed improvement regarding pain, function, and pinch strength. Laser therapy showed significantly better results compared to a wrist splint, but no significant difference was seen between high-power and LPL therapy groups. Nerve conduction evaluation findings did not reveal any significant difference.

Conclusion: Both the wrist splint and laser therapy can improve the symptoms of carpal tunnel syndrome. HPL therapy showed better results, although not significantly different from LPL therapy. Keywords: Carpal tunnel syndrome; High-intensity laser therapy; Low-intensity laser therapy; Electrodiagnostic evaluation.
\end{abstract}

\section{Introduction}

Carpal tunnel syndrome (CTS) is the most common peripheral nerve entrapment, which is caused by the median nerve compression in the wrist and affects millions around the world. ${ }^{1,2}$ The carpal tunnel consists of wrist bones and transverse ligaments (flexor retinaculum) in the anterior part of the wrist and the median nerve passes through this space with 9 tendons. Due to its specific position in the tunnel, the median nerve is highly prone to compression. ${ }^{3}$ The annual prevalence of CTS is estimated to be around $2.1 \%$ in the general population, ${ }^{4}$ with women more affected than men. ${ }^{5}$ Most patients complain of numbness, tingling, and pins and needles in the median nerve sensory innervation territory, which may get worse when sleeping at night. ${ }^{6,7}$ In the advanced stages of the disease, the atrophy of the thenar muscles occurs, accompanied by the weakness of the thumbs and index fingers, leading to the inability to hold objects by hand. ${ }^{8}$

This syndrome can be suspected in a patient according to history and physical examination, but the gold standard diagnostic method is using electrodiagnosis, which is vitally important in the diagnosis and assessment of the severity (which is necessary for choosing the

Please cite this article as follows: Hojjati F, Afjei MH, Ebrahimi Takamjani I, Rayegani SM, Sarrafzadeh J, Raeissadat SA, Payami S. The effect of high-power and low-power lasers on symptoms and the nerve conduction study in patients with carpal tunnel syndrome. A Prospective Randomized Single-Blind Clinical Trial. J Lasers Med Sci. 2020;11(suppl 1):S73-S79. doi:10.34172/jlms.2020.S12. 
treatment method) and is also helpful for ruling out other neuropathic disorders (such as radiculopathy, plexopathy or other peripheral nerve lesions. ${ }^{9}$

Many therapeutic methods have been suggested for CTS and there is no consensus on their superiority and the order of their use. ${ }^{10}$ Conservative treatments are the main therapeutic approaches in the first step. Conservative treatments include wrist splints, steroid injections in the carpal tunnel, anti-inflammatory medications, mobilization techniques, and physical modalities such as laser therapy, ultrasound and Bioptron. ${ }^{11-14}$ Carpal tunnel surgery is preserved for the severe involvement and the patients who are refractory to conservative therapy. ${ }^{15,16}$ Wrist splinting, as a method of avoiding further injury, is one of the most common treatments among CTS patients and has been endorsed by the American Academy of Orthopedic Surgeons (AAOS) clinical practice guideline published in 2009. ${ }^{17}$ Among various physical modalities, laser therapy and ultrasound methods may have potential effects on bringing about biophysical effects within tissues. $^{18}$

These results accelerate the wound healing process, as well as a significant reduction in pain, inflammation, and scarring in the tissues. The particles of laser energy are absorbed by a variety of molecules within the cell, a process that initiates several positive physiological responses. ${ }^{19}$ In essence, light energy is converted into biochemical energy. As a result, normal cell morphology and function are restored. The process is curative. ${ }^{20}$ Low-power laser (LPL) therapy is a non-invasive treatment method for many neuromusculoskeletal diseases. It is mainly believed that it can reduce pain and local inflammation by stimulating the micro vascularization and blocking pain enzymes and activating endorphins. ${ }^{21,22}$ Many studies have assessed the effect of LPL therapy on peripheral nerve injuries because it increases microcirculation in the tissue, promotes nerve function, increases the rate of axon growth and myelinization, and improves regeneration of the injured nerve. ${ }^{23}$ The high-power laser (HPL) is a technological advancement in the LPL field and can treat a much larger part of the body surface. Furthermore, it has deeper penetration and consumes less time compared with the LPL. The HPL or hot laser is 24 times more powerful than conventional lasers and its effective penetration depth is more than 4 centimeters, while LPL has an effective penetration depth of half a centimeter and can mainly help treat superficial and small tissues. ${ }^{24}$ The HPL is painless and safe and has no side effects. An increase in localized temperature can be observed in the skin due to laser penetration. ${ }^{25}$

Notwithstanding all these, laser therapy still faces many controversies, especially in neuromusculoskeletal disorders such as CTS. It is due to the lack of enough studies on this subject. To fill this lacuna, the present study aims to investigate and compare the effect of HPL on the CTS treatment process to determine whether HPL has moderate superiority over LPL therapy or splinting in relieving pain, grip strength, and electrodiagnosis in patients aged 30-50 years with mild and moderate CTS.

\section{Materials and Methods}

This is a prospective, single-blind clinical trial that was conducted in Shohada-e-Tajrish hospital between February and December 2018. This study was retrospectively registered at www.irct.ir with registry code: (ID:IRCT20201130049543N1).

\section{Patients}

The study population included all patients who came to the clinic of physical medicine and rehabilitation of Shohada-e-Tajrish hospital between February and December 2018 with signs and symptoms of CTS. The diagnosis of CTS was confirmed by electrodiagnostic studies (nerve conduction study and electromyography). Patients with confirmed mild or moderate CTS were selected. The patients were informed about the plan and goals of the study as well as its possible complications by a physician, and the informed consent form was completed by them. Inclusion criteria included patients from 30-50 years of age and the duration of symptoms for at least 3 months. Exclusion criteria included (1) The presence of other neuropathic diseases such as polyneuropathy, cervical radiculopathy or brachial plexopathy (including thoracic outlet syndrome), (2) Systemic diseases that can affect the severity or treatment, such as hypothyroidism, gout, systemic lupus erythematosus, rheumatoid arthritis, diabetes mellitus, chronic kidney deficiency, and acromegaly, (3) History of carpal tunnel release surgery, (4) Wrist fracture, (5) Pregnancy, (6) History of injection in the carpal tunnel in the last month.

\section{Randomization and Sampling}

A non-probability randomized (quota) sampling method was used. Forty-five patients with confirmed mild or moderate CTS were enrolled in the study, considering their willingness to enter the study. Then 15 patients were randomly assigned to the control group, 15 patients to the LPL group, and 15 patients to the HPL group.

\section{Blinding}

This is a single-blind study. A physician (specialist in physical medicine and rehabilitation) who was blinded to the patient's group was in charge of the electrodiagnostic assessment and collecting data of all patients (including demographic data, visual analog scale [VAS], Boston questionnaire, grip strength, and electrodiagnosis findings). Moreover, he gave instructions about using the wrist splint to all participants. A separate physician did the HPL therapy and a therapist did the low-level laser therapy, both blinded to patients' assessment. However, due to this limitation that we did not use a sham laser, the patients could not be blinded, although the patients in 
both laser therapy groups were blinded about the power of the laser emission they received.

\section{Intervention}

During the treatment, the patients did not use any medication, and care instructions were equally provided to the groups. The patients were randomly assigned to one of the treatment groups: wrist splint (control group), wrist splint + LPL, and wrist splint + HPL. A wrist splint with a metal bar was used at an angle of $0^{\circ}$ for 12 weeks in each of the three treatment groups. The patients were instructed to wear the splint during the night and as long as they could during the day time. In the LPL group, the patients underwent LPL radiation using a Fisioline, Lumix 2 device ( $45 \mathrm{~W}$ ) with a wavelength of $775 \mathrm{~nm}$, a frequency of $6500 \mathrm{~Hz}$, and an intensity of $20 \mathrm{~J} / \mathrm{cm}^{2}$ on the carpal tunnel region (along with the flexor retinaculum, just distal to wrist palmar crease). In the HPL group, the laser was applied using the BTL-6000 device, with a wavelength of $1064 \mathrm{~nm}$, an intensity of $20 \mathrm{~J} / \mathrm{cm}^{2}$, and 5 -W power, for 36 seconds, to the same anatomic region similar to the LPL group. No topical lotion or ointment was used during laser therapy. Eye protection glasses were used during laser therapy by both the patient and the physician. The results were evaluated immediately and 12 weeks after the treatment.

\section{Data Collection}

The numeric VAS was used for pain assessment, ranging from 0 (No pain) to 10 (the most severe pain imaginable). Pinch strength was measured by a manual dynamometer (Hydraulic pinch gauge, Saehan Company, South Korea). The patient was in a comfortable sitting position during the measurement and the mean force generated in 3 attempts was calculated and recorded. The electrodiagnostic evaluation was done using the UltraPro S100 device (Natus Company, Denmark), including the assessment of median nerve sensory action potential (sensory nerve action potential or SNAP) and motor action potential (compound motor action potential or CMAP). SNAP peak latency (PL) and amplitude (amp) and CMAP onset latency (OL), amplitude, and nerve conduction velocity (NCV) were measured and recorded for each patient. The severity of symptoms and the functional status of the patients were evaluated using the Boston questionnaire. The Boston questionnaire consists of two parts: measuring the severity of symptoms and measuring the patient's functional status. BQ-SS: Boston Symptom Severity Scale (BQ-SS) consists of 11 questions about the severity and frequency of symptoms, including night and day numbness, pins and needles, pain, and muscle weakness. BQ-FS: Boston Questionnaire Functional Scale (BQ-FS) consists of 8 questions concerning the patient's problems in performing specific activities such as writing, holding a book, buttoning up the shirt, holding the phone, opening jam jar, doing hard house chores, taking a bath, carrying a shopping bag, and dressing. Each question was answered using a 5-point Likert scale that consists of 5 options and each option is assigned a score ranging from 1 to 5 , with scores 1 indicating no symptoms and 5 indicating the most severe symptoms. To determine the severity of symptoms and functional status, the mean scores were calculated in each part. The higher the mean score, the greater the severity of the symptoms, and the disability of the patient would be. Thus, the patients were divided into 5 groups based on the score obtained: 1 . healthy (0.1-1), 2. mild (1.1-2), 3. moderate (2.1-3), 4. severe involvement (3.1-4), and 5. very severe involvement (4.1-5).

Individuals who were literate enough filled out a questionnaire by themselves, but the questionnaire was read face to face for those people with lower literacy and reading difficulties.

All of the variables were measured before the treatment and immediately and 12 weeks after the last treatment session.

\section{Data Analysis}

All documented information was analyzed using SPSS version 21 and appropriate statistical tests were used for data analysis. A $P$ value less than 0.05 was considered as statistically significant.

\section{Results}

A total number of 63 patients were eligible for the study, but 13 were excluded (mainly due to the presence of radiculopathy), 2 did not meet the inclusion criteria (they had severe CTS) and 3 refused to participate. Finally, 45 patients were enrolled (Figure 1). Demographic data were collected and compared between groups. All patients were women. The patients' mean age, height, and weight in the control group were $46.9 \pm 1.7$ years, $159.1 \pm 1.9$ $\mathrm{cm}$, and $65 \pm 2.6 \mathrm{~kg}$ respectively. The patients' mean age, height, and weight in the LPL group were $48.7 \pm 1.9$ years, $159.6 \pm 2.22 \mathrm{~cm}$, and $61 \pm 2.4 \mathrm{~kg}$ respectively. The patients' mean age, height, and weight in the HPL group were 46.9 \pm 1.7 years, $159 \pm 2.06 \mathrm{~cm}$, and $63 \pm 2.7 \mathrm{~kg}$ respectively. There was no significant difference between the 3 groups. $P$ value $<0.001$ was considered statistically significant. Moreover, there was no significant difference between the 3 groups in terms of mean pinch strength, pain severity (VAS and BQ-SS), functional status (BQ-FS), and the electrodiagnostic parameters before the therapeutic intervention (Table 1).

In terms of pinch strength, all groups showed improvement in the follow-up sessions, both immediately and 12 weeks after the treatment; the change in the LPL and HPL groups was significant at both follow-up sessions $(P<0.001)$, but in the control group there were no significant changes seen immediately $(P=0.061)$ and 12 weeks $(P=0.091)$ after the treatment. The highest mean pinch strength belonged to the HPL group, with a mean of $11.93 \mathrm{~kg}$ immediately and $12.12 \mathrm{~kg} 12$ weeks after 


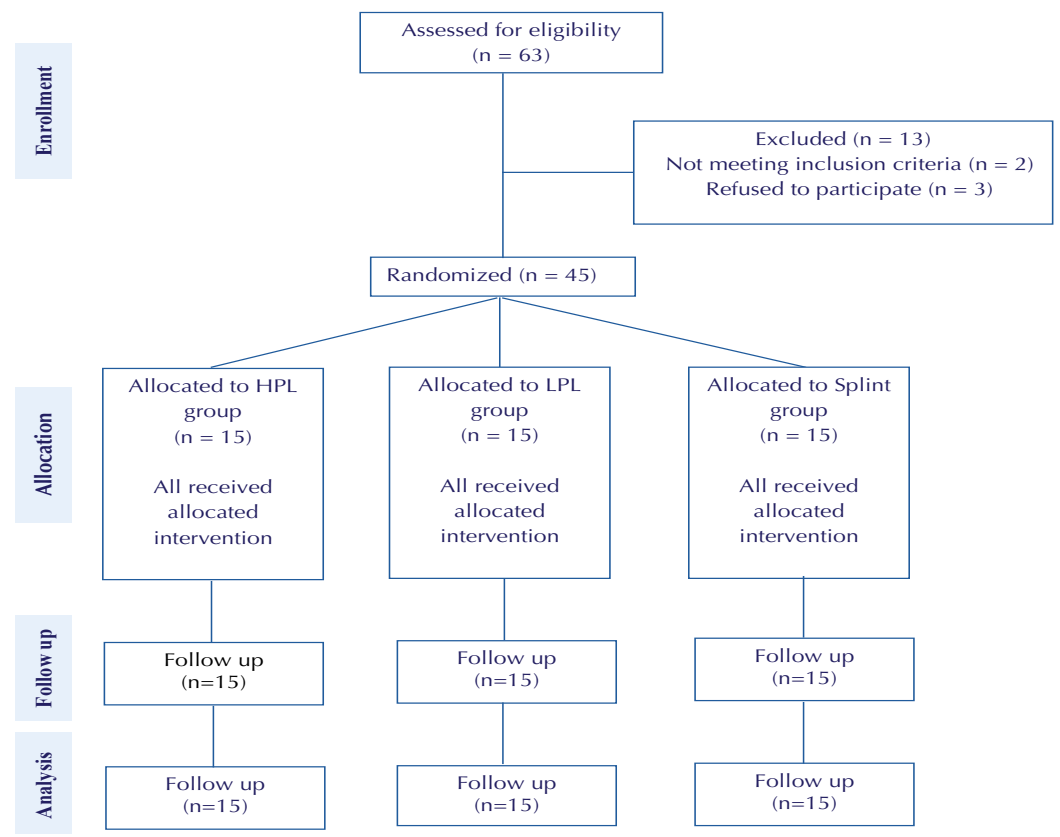

Figure 1. Consort table

the treatment; and the lowest mean strength was seen in the control group immediately and 12 weeks after the treatment, with a mean strength of $9.54 \mathrm{~kg}$ and $9.75 \mathrm{~kg}$ respectively (Figure 2).

The evaluation of VAS score changes immediately and 12 weeks after the treatment period showed a significant decrement in all groups $(P<0.001)$ compared to the base evaluation, but there was no significant difference between the groups $(P=0.050)$. Patients in the HPL group had the lowest VAS scores, with a mean of 4.12 immediately after treatment and 3.2, 12 weeks after the treatment; the highest scores were found in the control group with means of 7.46 and 6.73 immediately and 12

Table 1. Demographic and evaluational parameters difference between groups before treatment.

\begin{tabular}{lc}
\hline Variable & P value \\
\hline Age & 0.645 \\
Height & 0.945 \\
Weight & 0.051 \\
Before treatment & \\
Mean pinch strength & 0.096 \\
VAS & 0.200 \\
BQ-SS & 0.873 \\
BQ-FS & 0.341 \\
SNAP PL & 0.168 \\
SNAP amp & 0.996 \\
CMAP OL & 0.298 \\
CMAP amp & 0.976 \\
CMAP NCV & 0.984 \\
\hline
\end{tabular}

weeks after the treatment respectively (Figure 3).

In terms of the symptoms severity scale, all three groups showed a significant improvement immediately and 12 weeks after the treatment $(P<0.001)$, but there was no significant difference between the two groups $(P=0.123)$. The lowest score immediately after the treatment belonged to the HPL group (mean: 24), but 12 weeks after the treatment the lowest score was seen in the LPL group (mean:17) (Figure 4).

Assessing the functional status immediately and 12 weeks after the treatment indicated a significant improvement in all groups $(P<0.001)$, with the lowest score (better function) in the HPL group (13.2 and 10.2 respectively). There was no significant difference between the LPL and HPL groups $(P=0.164)$. The minimum changes were seen in the control group, especially in the comparison between the 2 follow-up sessions (Figure 5).

Assessing the electrodiagnostic parameters, including sensory peak latency (SNAP PL), sensory amplitude (SNAP amp), motor onset latency (CMAP OL), motor amplitude (CMAP amp), and CMAP NCV, is provided below in turn. All numbers were rounded to one decimal place for easier evaluation.

In terms of SNAP PL, all groups showed a very small decrement immediately and 12 weeks after the treatment, but none of them showed a significant improvement $(P=0.123)$ (Figure 6).

Assessing SNAP amp changes revealed no significant changes in all three groups $(P=0.624)$ (Figure 7).

Changes in CMAP OL were seen in all groups, with no significant differences. The changes did not indicate a certain trend; as it is seen in the HPL group, there was a minimal decrement immediately after the treatment but 


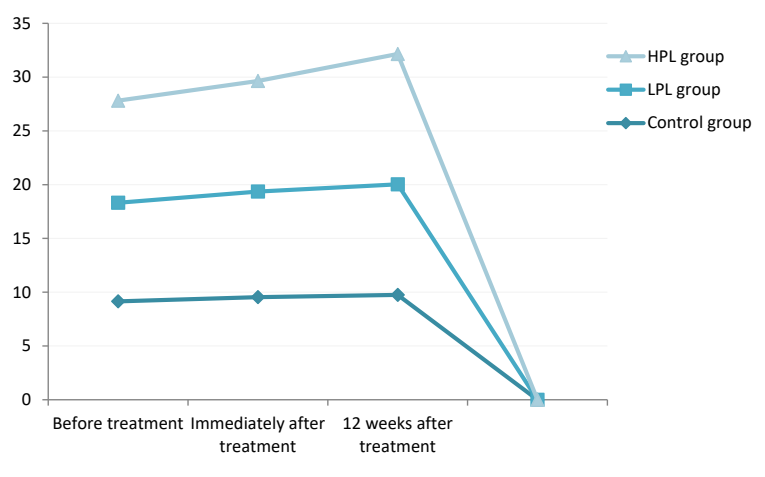

Figure 2. Mean Pinch Strength.

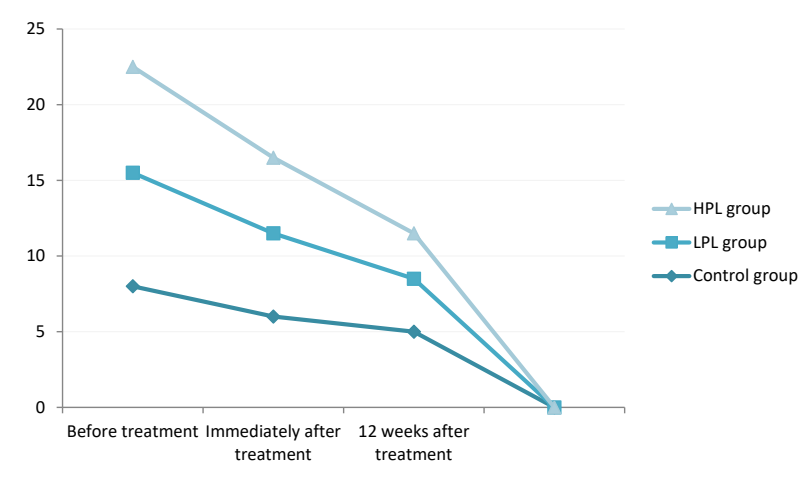

Figure 3. VAS Score.

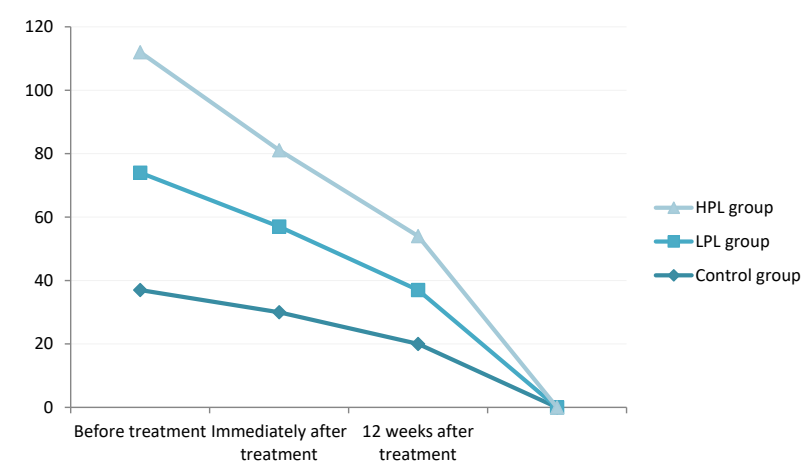

Figure 4. Symptom Severity Scale Score (BQ-SS).

again an increment after 12 weeks (Figure 8).

The evaluation of CMAP amplitude showed changes in all three groups but not statistically significant $(P=0.164)$. The highest CMAP amp immediately and 12 weeks after the treatment was seen in the HPL group (7.5 and $7.7 \mathrm{mV}$ respectively) (Figure 9).

Finally, CMAP NCV evaluation showed no certain trend, with no significant changes in any groups $(P=0.164)$. However, minimal improvement was seen in the LPL and HPL groups. The highest NCV was seen in the HPL group immediately $(55.81 \mathrm{~m} / \mathrm{s})$ and 12 weeks $(56.12 \mathrm{~m} / \mathrm{s})$ after the treatment (Figure 10).

\section{Discussion}

The purpose of this study was to compare the efficacy of low-power and HPL therapies on pain, function, and electrodiagnostic parameters in patients with mild or moderate CTS. For this purpose, the patients were randomly divided into three groups (control, LPL, and HPL groups).

In the first stage, the patients' age, height, and weight were measured and recorded, which showed no significant difference between the groups. Moreover, VAS, symptom severity scale, functional scale, pinch grip, and electrodiagnostic parameters were evaluated before the treatment (basis), immediately after the treatment (first follow-up session) and 12 weeks after (second follow-up session) the treatment. There was no significant difference regarding these evaluation findings between the three groups at the baseline assessment. The results were compared between the first and second follow-up sessions relative to each other and to the baseline assessment. The results were also compared between different groups.

As mentioned in the results section, the pain decreased in all 3 groups according to VAS and BQSS evaluation, but there was no significant difference between the groups. The pain decrement could be due to wrist immobility, using the wrist splint in all groups. However, more decrement was seen in the laser therapy groups, which can propose the role of laser energy in decreasing pain, although no significant decrement was seen. Moreover, no significant difference was seen between the LPL and HPL groups, which can be due to

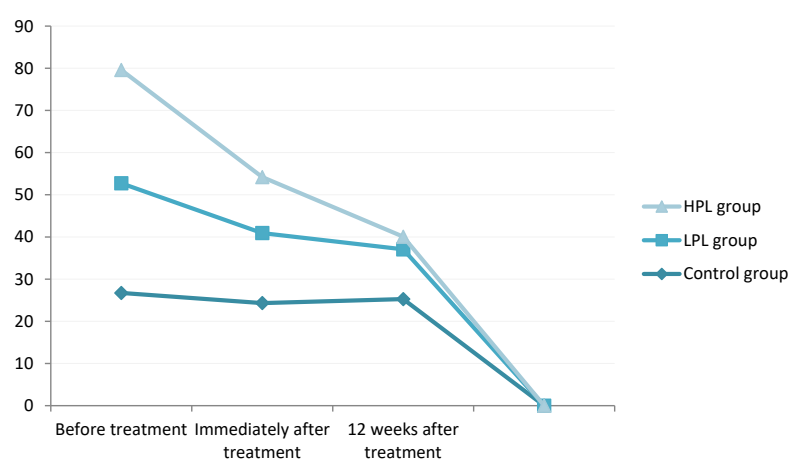

Figure 5. Functional Status (BQ-FS).

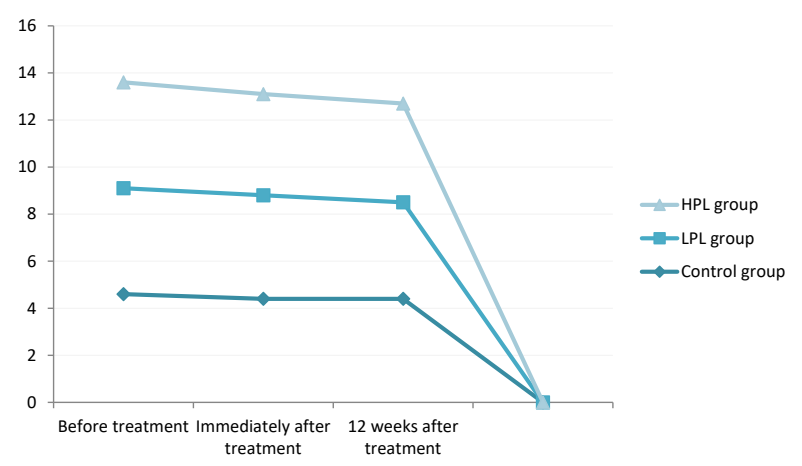

Figure 6. Sensory peak latency 


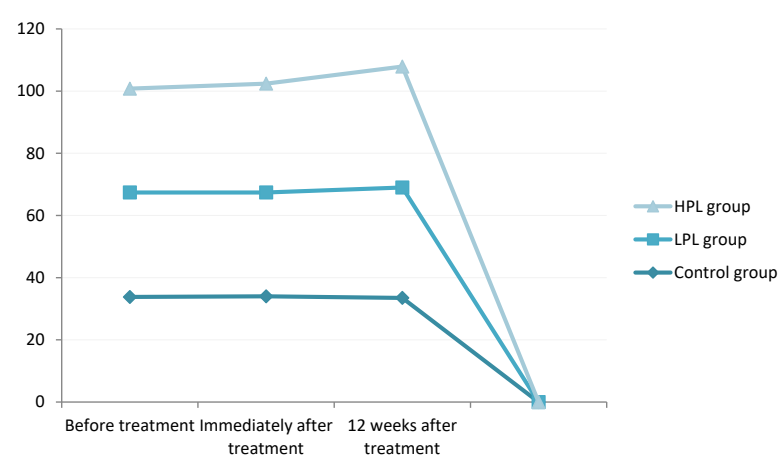

Figure 7 . Sensory amplitude

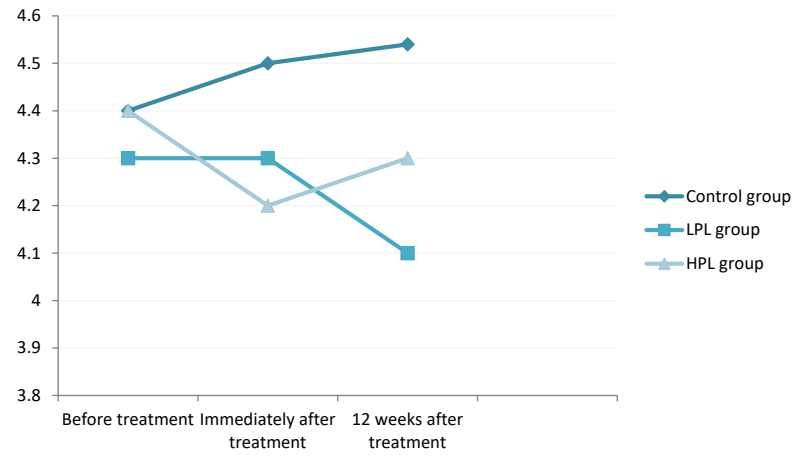

Figure 8. Motor Onset Latency.

the almost equal total energy radiated in both groups. Our study also showed functional improvement in all three groups but no significant difference between the groups. This should be noted that an improvement in function can be a direct result of pain decrement, so it looks logical that by decreasing the pain the function improves. In line with our study results, Rayegani et $\mathrm{al}^{8}$ showed that low-level laser therapy resulted in a pain decrement and functional improvement in 50 patients with mild and moderate CTS. They also mentioned an improvement in electrodiagnostic parameters, although with no significance. In our study, the evaluation of the electrodiagnostic parameters showed variable results. This can most likely be due to technical errors and intrarater reliability, which are common in electrodiagnostic studies, although with no clinical significance in most cases. The results of Casale and colleagues' study showed that positive and negative sensory symptoms improved by HPL therapy )a combination of the $830 \mathrm{~nm}$ and 1064 $\mathrm{nm}$ laser wavelengths), leading to decreased pain, itch sensation as well as improvement in neuropsychological parameters. Moreover, their results showed better improvement than transcutaneous nerve electrical stimulation, but there was no statistically significant difference between these modalities. ${ }^{24}$

In our study, the HPL group showed more improvement in pinch strength, VAS, symptom severity scale (immediately after treatment), and functional status, but the difference was not significant compared to the LPL group. Both low-power and HPL therapy groups showed better results than the control group, in which improvement was seen, confirming the effect of wrist immobilizing and rest on CTS symptoms. Various studies have demonstrated that lasers can be effective in reducing pain. Reducing pain results in better function which is approved in our study. Moreover, we hope by using lasers we can see tissue repair, especially neural tissue in nerve injury cases such as CTS. Neural tissue improvement needs to be approved by electrodiagnostic studies, not only by a reduction in symptoms. We should remind that there are few studies on HPL therapy in the field of neuromuscular disorder treatment, because of which laser therapy parameter selection is still controversial. We decided to have total laser energy near to LPL therapy to keep the patients safe. This can have a major impact on the results of the study. More studies are needed on the high power-laser effects on human tissues, so we can write more confidently about its effects on various tissues, including neural tissue.

\section{Conclusion}

Both the wrist splint and laser therapy can improve the symptoms of CTS, leading to better function. HPL therapy showed better results, although not significantly different from LPL therapy.

\section{Ethical Considerations}

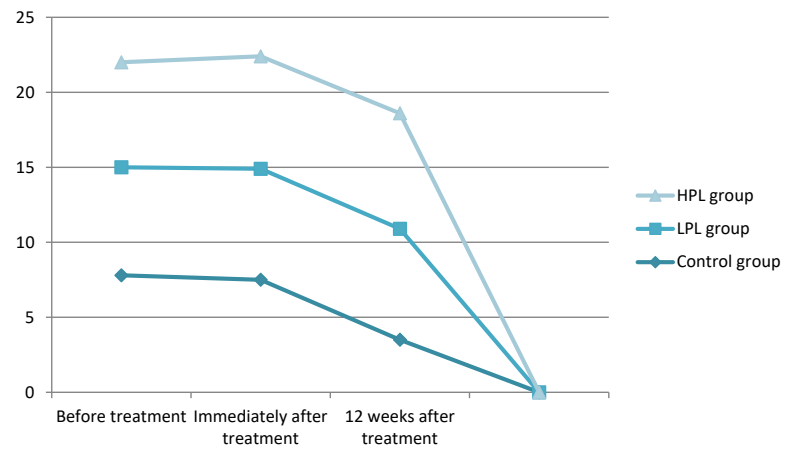

Figure 9. Motor Amplitude.

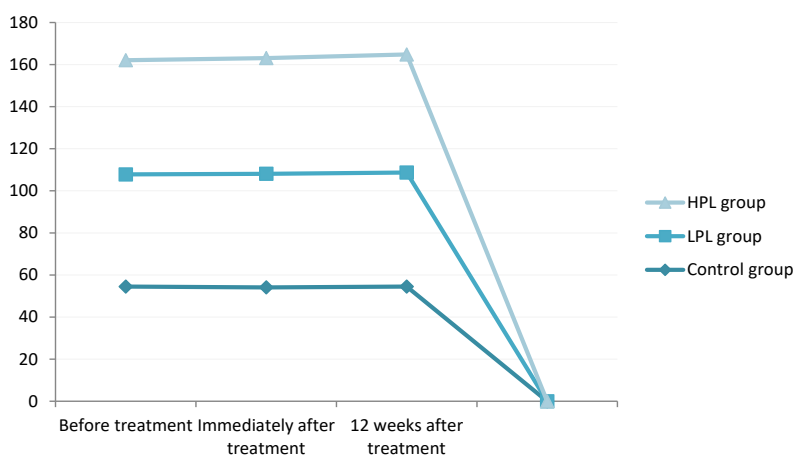

Figure 10. Motor Nerve Conduction Velocity Changes. 
This study has been approved by the Medical Ethics Committee of the Faculty of Rehabilitation Sciences of Iran (IR.IUMS.REC1396 9211342201).

\section{Conflict of Interests}

The authors declare that there is no conflict of interest regarding the publication of this article.

\section{References}

1. Rayegani SM, Sedighipour L, Raeissadat SA, Hojjati F, Aghayousefi HR, Lahiji F, et al. Co-occurrence of trifid and bifid median nerves in a patient with bilateral carpal tunnel syndrome. Neurol India. 2015;63(5):788-9. doi: 10.4103/0028-3886.166535.

2. Torpy JM, Lynm C, Golub RM. Carpal Tunnel Syndrome. JAMA. 2011;306(20):2283. doi: 10.1001/jama.306.20.2283.

3. Pritsch T, Rosenblatt Y, Carmel A. [Carpal tunnel syndrome]. Harefuah. 2004;143(10):743-748, 764, 765. [In Hebrew]

4. El Miedany Y, Ashour S, Youssef S, Mehanna A, Meky FA. Clinical diagnosis of carpal tunnel syndrome: old tests-new concepts. Joint Bone Spine. 2008;75(4):451-7. doi: 10.1016/j. jbspin.2007.09.014.

5. Love C. Carpal tunnel syndrome. J Orthop Nurs. 2003;7(1):33-42. doi: 10.1016/S1361-3111(02)00124-3.

6. Palmer BN. Carpal tunnel syndrome, active component, U.S. armed forces, 2000-2010. MSMR. 2011;18(7):12-5.

7. Field T, Diego M, Cullen C, Hartshorn K, Gruskin A, Hernandez-Reif $\mathrm{M}$, et al. Carpal tunnel syndrome symptoms are lessened following massage therapy. $J$ Bodyw Mov Ther. 2004;8(1):9-14. doi:10.1016/S13608592(03)00064-0.

8. Rayegani SM, Bahrami MH, Eliaspour D, Raeissadat SA, Shafi Tabar Samakoosh M, Sedihgipour L, et al. The effects of low intensity laser on clinical and electrophysiological parameters of carpal tunnel syndrome. J Lasers Med Sci. 2013;4(4):182-9.

9. Kozak A, Schedlbauer G, Wirth T, Euler U, Westermann C, Nienhaus A. Association between work-related biomechanical risk factors and the occurrence of carpal tunnel syndrome: an overview of systematic reviews and a meta-analysis of current research. BMC Musculoskelet Disord. 2015;16:231. doi: 10.1186/s12891-015-0685-0.

10. Somaiah Aroori RAS. Carpal tunnel syndrome. Ulster Med J. 77(1):6-17.

11. Wright TW, Glowczewskie F, Wheeler D, Miller G, Cowin D. Excursion and strain of the median nerve. J Bone Joint Surg Am. 1996;78(12):1897-903. doi: 10.2106/00004623199612000-00013.

12. Hough AD, Moore AP, Jones MP. Reduced longitudinal excursion of the median nerve in carpal tunnel syndrome. Arch Phys Med Rehabil. 2007;88(5):569-76. doi: 10.1016/j. apmr.2007.02.015.

13. Coppieters MW, Alshami AM. Longitudinal excursion and strain in the median nerve during novel nerve gliding exercises for carpal tunnel syndrome. J Orthop Res. 2007;25(7):972-80. doi: 10.1002/jor.20310.

14. Ettema AM, Zhao C, Amadio PC, O’Byrne MM, An KN. Gliding characteristics of flexor tendon and tenosynovium in carpal tunnel syndrome: a pilot study. Clin Anat. 2007;20(3):292-9. doi: 10.1002/ca.20379.

15. Dawson DM. Entrapment neuropathies of the upper extremities. N Engl J Med. 1993;329(27):2013-8. doi: 10.1056/NEJM199312303292707.

16. Karimzadeh A, Bagheri S, Raeissadat SA, Bagheri S, Rayegani SM, Rahimi-Dehgolan S, et al. The comparison of the effectiveness between different doses of local methylprednisolone injection versus triamcinolone in carpal tunnel syndrome: A double-blind clinical trial. J Pain Res. 2019;12:579-84. doi: 10.2147/JPR.S190652.

17. Bahrami MH, Raeissadat SA, Nezamabadi M, Hojjati F, Rahimi-Dehgolan S. Interesting effectiveness of ozone injection for carpal tunnel syndrome treatment: a randomized controlled trial. Orthop Res Rev. 2019;11:61-7. doi: 10.2147/ORR.S202780.

18. Rayegani SM, Moradi-Joo M, Raeissadat SA, Bahrami MH, Seyed-Nezhad M, Heidari S. Effectiveness of low-level laser therapy compared to ultrasound in patients with carpal tunnel syndrome: A systematic review and meta-analysis. J Lasers Med Sci. 2019;10(suppl 1): S82-S89. doi: 10.15171/ jlms.2019.S15.

19. Rayegani SM, Bayaat M, Sedighipour L, Samadi B. Low intensity laser therapy. J Lasers Med Sci. 2010;1(1):31-4.

20. Greenberg DA, Aminoff MJ, Simon RP. Clinical Neurology. 8th ed. New York: McGraw-Hill Medical; 2012.

21. Walker JB, Akhanjee LK. Laser-induced somatosensory evoked potentials: evidence of photosensitivity in peripheral nerves. Brain Res. 1985;344(2):281-5. doi: 10.1016/0006-8993(85)90805-4.

22. Viera Aleman C, Puron E, Hamilton ML, Santos Anzorandia C, Navarro A, Pineda Ortiz I. Evaluation of motor and sensory neuroconduction of the median nerve in patients with carpal tunnel syndrome treated with noncoherent light emitted by gallium arsenic diodes. Rev Neurol. 2001;32(8):717-20. [In Spanish].

23. Yamany AA, Sayed HM. Effect of low level laser therapy on neurovascular function of diabetic peripheral neuropathy. $J$ Adv Res. 2012;3(1):21-8. doi: 10.1016/j.jare.2011.02.009.

24. Casale R, Damiani C, Maestri R, Wells CD. Pain and electrophysiological parameters are improved by combined 830-1064 high-intensity LASER in symptomatic carpal tunnel syndrome versus transcutaneous electrical nerve stimulation. A randomized controlled study. Eur J Phys Rehabil Med. 2013;49(2):205-11.

25. Song HJ, Seo HJ, Lee Y, Kim SK. Effectiveness of highintensity laser therapy in the treatment of musculoskeletal disorders: A systematic review and meta-analysis of randomized controlled trials. Medicine (Baltimore). 2018;97(51):e13126. doi: 10.1097/MD.0000000000013126. 